\title{
Real-time parameter estimation of Zika outbreaks using model averaging
}

C. R. SEBRANGO-RODRÍGUEZ ${ }^{1,2} \dagger$, D. A. MARTÍNEZ-BELLO ${ }^{3}{ }^{*}$, L. SÁNCHEZ-VALDÉS $S^{1,4}$, P. J. THILAKARATHNE ${ }^{5}$, E. DEL FAVA ${ }^{6}$, P. VAN DER STUYFT ${ }^{7,8}$, A. LÓPEZ-QUÍLEZ ${ }^{3}$ AND Z. SHKEDY ${ }^{2}$

${ }^{1}$ University of Sancti Spiritus 'José Martí Pérez', Avenida de los Martires 360, Sancti Spiritus, Cuba

${ }^{2}$ Interuniversity Institute for Biostatistics and Statistical Bioinformatics (I-BioStat), Hasselt University, Agoralaan - Building D, 3590 Diepenbeek, Belgium

${ }^{3}$ Department of Statistics and Operations Research, Faculty of Mathematics, Universitat de València, C/Dr. Moliner, 50, 46100 Burjassot, València, Spain

${ }^{4}$ Centro de Inmunología Molecular, Calle 16 esq.15 Atabey, Playa, Ciudad de La Habana

${ }^{5}$ Interuniversity Institute for Biostatistics and Statistical Bioinformatics, Katholieke Universiteit Leuven, Kapucijnenvoer 35, Blok D, Box 7001, B3000, Leuven, Belgium

${ }^{6}$ Carlo F. Dondena Centre for Research on Social Dynamics, Bocconi University, Via Guglielmo Rontgen 1, 20136 Milan, Italy

${ }^{7}$ Unit of General Epidemiology and Disease Control, Institute of Tropical Medicine, Antwerp, Belgium

${ }^{8}$ Department of Public Health, Ghent University, Ghent, Belgium

Received 17 December 2016; Final revision 17 March 2017; Accepted 28 April 2017; first published online 1 June 2017

\section{SUMMARY}

Early prediction of the final size of any epidemic and in particular for Zika disease outbreaks can be useful for health authorities in order to plan the response to the outbreak. The Richards model is often been used to estimate epidemiological parameters for arboviral diseases based on the reported cumulative cases in single- and multi-wave outbreaks. However, other non-linear models can also fit the data as well. Typically, one follows the so called post selection estimation procedure, i.e., selects the best fitting model out of the set of candidate models and ignores the model uncertainty in both estimation and inference since these procedures are based on a single model. In this paper we focus on the estimation of the final size and the turning point of the epidemic and conduct a real-time prediction for the final size of the outbreak using several nonlinear models in which these parameters are estimated via model averaging. The proposed method is applied to Zika outbreak data in four cities from Colombia, during the outbreak ocurred in 2015-2016.

Key words: Five-parameter logistic, four-parameter Gompertz, Richards, three-parameter logistic, Weibull.

\footnotetext{
* Author for correspondence: D. A. Martínez-Bello, Department of Statistics and Operations Research, Faculty of Mathematics, Universitat de València, C/Dr. Moliner, 50, 46100 Burjassot, València, Spain.

(Email: danieladyro@gmail.com)

$\dagger$ These authors contributed equally to this work.
}

\section{INTRODUCTION}

Zika infection is an arboviral disease characterized by subclinical or mild dengue-like illness, with severe manifestations such as Guillain-Barre syndrome in adults and microcephaly in babies born to infected mothers [1]. During 2015 and 2016 the disease affected several 
South-American countries, especially Brazil and Colombia. In Colombia, a total number of 104755 cases were recorded, from which 8826, 92113 , and 3816 cases were confirmed by laboratory, diagnosed by clinical symptoms, and suspected without confirmation, respectively. Zika cases were recorded between the 32th epidemiological week, 2015 until the 42nd epidemiological week, 2016 (09/08/2015-22/10/2016) [2]. For the analysis presented in this paper we use the data from four cities, Bucaramanga (3651 cases), Cali (12 220), Cúcuta (4287), and Neiva (1940).

Mathematical and statistical models are increasingly being used to facilitate the estimation of the primary epidemiological parameters in infectious disease outbreak. During a single-peak outbreak, the turning point (or primary inflection point), i.e. the point in time at which the rate of accumulation changes from increasing to decreasing, and the final size of the epidemic are among the epidemiological parameters of interest to be estimated [3-5]. Once an outbreak has begun, knowledge about the potential severity in realtime (i.e. before the end of the outbreak) can help public health authorities to respond effectively [5].

Various epidemiological studies used mathematical and statistical models to describe the evolution and spread of severe acute respiratory syndrome (SARS) and dengue and to evaluate the impact of control interventions. In particular, Hsieh et al. [3, 5] and Zhou et al. [4] proposed to use a non-linear model, the Richards model [6], in order to model the cumulative number of reported cases, to estimate the turning point and the basic reproduction number, $R_{0}$. Hsieh et al. applied non-linear models to model epidemics of SARS [7], dengue disease [8-10], Influenza A (H1N1) [11] and Ebola [12]. In addition to the estimation of the epidemiological parameters, Hsieh et al. [3] used the model for real-time prediction of these parameters for dengue and SARS outbreaks.

Hsieh et al. estimation and prediction is based on the Richards model and considers only the cumulative infective population size with saturation in growth as the outbreak progresses. The basic premise of the Richards model is that the incidence curve consists of a single peak of high incidence, resulting in an $S$-shaped epidemic curve and a single turning point of the outbreak [7, 8]. Hsieh et al. [7, 8] also showed that the Richards model can be used to model multiwave outbreaks as well. In this paper we focused on a single-wave outbreak.

A variety of non-linear models have been developed to model growth data. Among them, we consider the three-parameter logistic (3P logistic) [7, 13, 14], five-parameter logistic (5P logistic) [15], Sigmoid Emax [16], Gompertz [14, 17], and Weibull [18] models. All these models can be used to fit epidemic data as well. Fitting several models to the same data raises the issue, central in statistical modeling, of model selection. Indeed, a model selection procedure is needed in order to choose the model with the best fit to the data. Often, one is confronted with the problem that several models are performing almost equally well over the range of observed data. Typically, one selects the best-fitting model out of the set of fitted models and ignores the uncertainty due to model selection in estimation and inference. For these reasons, several authors (i.e. Burnham \& Anderson [19], Claeskens \& Hjort [20], Posada [21], Moon [22], and Lin [23]), advocate the use of model averaging (MA) techniques to perform multimodel estimation and inference. MA is a method that takes into account all fitted models for the estimation of the parameters of primary interest. It is based upon a weighted average of the parameter of primary interest obtained from different models, giving largest weights to those models that best fit the data [24].

In the current paper we analyse Zika outbreak data and estimate a model average of the final size and the turning point of the epidemic, and perform a real-time prediction using several non-linear models. A realtime prediction is a procedure in which the final size of the outbreak is estimated as early as possible. An elaborate description of the procedure is given in the supplementary material for the paper (Supplementary Fig. S1). The proposed method is applied to four Zika outbreaks that occurred in four cities in Colombia during the 2015/2016 outbreak.

\section{DATA}

The data used in this paper were collected from four cities from Colombia, where Zika disease cases were reported to the Instituto Nacional de Salud (Colombian National Institute of Health) in a weekly basis. The study locations represent cities with the highest number of Zika cases within all the cities in Colombia. Two of the cities are located to the northeast of Colombia (Bucaramanga and Cúcuta) and two are located to the southwest (Cali, Neiva).

The weekly counts of clinical Zika cases by date of onset of symptom per city were converted into cumulative case curves starting on the 50 epidemiologic week (EW) of 2015 for Cali and Bucaramanga, and 
Table 1. Epidemiological information on the 2015/2016 Zika outbreak in the four cities from Colombia

\begin{tabular}{|c|c|c|c|c|c|c|c|}
\hline Area & Population & $\begin{array}{l}\text { Elevation } \\
\text { (meters) }\end{array}$ & Start (2015) & End (2016) & $\begin{array}{l}\text { Outbreak } \\
\text { length } \\
\text { (EW) }\end{array}$ & $\begin{array}{l}\text { Observed } \\
\text { final size }\end{array}$ & $\begin{array}{l}\text { Attack rate } \\
\text { per } 10000 \\
\text { inhabitants }\end{array}$ \\
\hline Bucaramanga & 528269 & 959 & 13 December & 10 September & 39 & 3651 & $69 \cdot 11$ \\
\hline Cali & 2394925 & 1000 & 13 December & 10 September & 39 & 12220 & $51 \cdot 02$ \\
\hline Cúcuta & 656380 & 320 & 22 November & 2 April & 19 & 4287 & $65 \cdot 31$ \\
\hline Neiva & 344026 & 442 & 22 November & 2 April & 19 & 1940 & $56 \cdot 39$ \\
\hline
\end{tabular}

the 47 EW for Cucuta and Neiva. The starting and ending dates of the outbreaks, as well as the observed number of cases at the end and the observed attack rates are shown in Table 1.

Figure 1 shows incidence and cumulative number of cases for the four cities under study. The outbreaks in Cúcuta and Neiva started and ended earlier (47 EW 2015 until 13 EW 2016) than the outbreaks in Cali and Bucaramanga (50 EW 2015 until 36 EW 2016).

\section{METHODS}

\section{Modeling Zika outbreak using non-linear models}

The Richards model [6, 25, 26] has often been used to model reported cumulative cases in disease outbreaks. In particular, Hsieh et al. [5, 7] demonstrated that Richards model can be used for real-time prediction of outbreak severity by estimating the carrying capacity (i.e.the final size of the epidemic) as well as realtime detection of the turning point (i.e. the time point with the peak number of cases) of the epidemic.

In Hsieh et al. [5-10], the estimates were obtained under the assumption that the cumulative number of reported cases at time $t, Y_{t}$, are normally distributed with mean $\mu(t, \theta)$ and variance $\sigma^{2}, Y_{t} \sim N\left(\mu(t, \theta), \sigma^{2}\right)$. The mean structure for $\mu(t, \theta)$ is given in the first line in Table 2. As pointed out by Hsieh et al. [5-9] the parameter vector to be estimated is $\theta=(\alpha, \gamma, k, \eta)$, where $\alpha$ is the final size of the epidemic, $\gamma$ is the per capita intrinsic growth rate of the infected population, $k$ is the exponent of the deviation from the standard logistic curve and $\eta$ is the turning point.

The cumulative number of reported cases in a Zika outbreak is an example of growth data. For many types of growth data, the growth rate does not steadily decline, but rather increases to a maximum before steadily declining to zero. In such models, $\eta$ is the position of the point of inflection (turning point of the epidemic for the application presented in this paper), the time when the growth rate is greatest.
Table 2 presents other five possible non-linear models. Note that all these models are scaled in calendar time (for which $t=1$ is the first epidemiological week in which the outbreak ocurred). The 3P logistic model [13, 14] is a special case of the Richards model, obtained when the exponent $k=1$. For the 3P logistic model, the growth curve is symmetric around turning point and has equal periods of slow and fast growth. The Gompertz model [14, 17] is another special case of the Richards function when $\gamma \rightarrow 0$ and is frequently used in situations where growth is not symmetrical about the turning point. There are many variants of the Weibull model, the one we use in this paper is a modification of the Gompertz model when its independent variable, time, is rescaled by logarithmic transformation [18]. Note that for all the models in Table 2 the turning point and the final size of the epidemic are parameters in the model.

The sigmoid Emax model and the 5P logistic are commonly used in dose-response modeling [23]. The sigmoid Emax model [16] is obtained by mathematical transformation of 3P logistic model and rescaling the independent variable by a logarithmic transformation. Similarly, the 5P logistic model is obtained by rescaling the independent variable by logarithmic transformation and by doing a reparametrization, so that the model evaluated at the inflection point $(\eta)$ reaches $50 \%$ of maximum response [15].

The first derivative of $\mu(t, \theta), \mu^{\prime}(t, \theta)=(\partial \mu(t, \theta) / \partial t)$, is the number of cases at time $t$, i.e., the incidence at time $t$. Except for the 3P logistic model, $\mu^{\prime}(t, \theta)$ is not symmetric around the turning point.

\section{Model uncertainty, model selection, and MA}

In the previous section, we presented six non-linear models that can be used for the estimation of epidemiological parameters and for real-time prediction. In this section, we describe the MA technique 

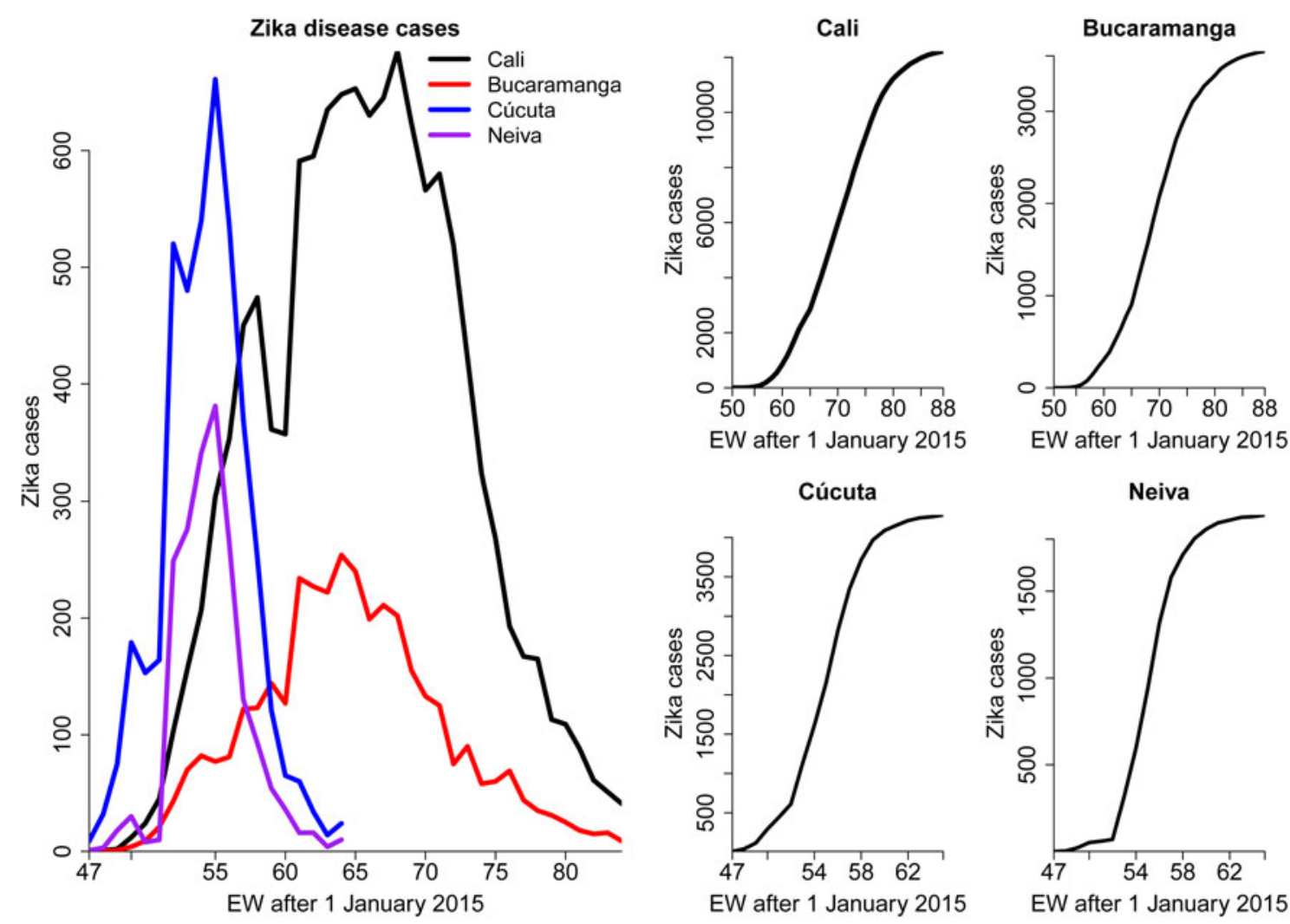

Fig. 1. Weekly number of cases (left) and cumulative cases (right) of Zika disease for the 2015/2016 outbreak in four cities from Colombia.The time scale is given in EW.

Table 2. Non-linear models considered to fit the cumulative cases of Zika outbreak

\begin{tabular}{llc}
\hline \hline Models & $\mu(t, \theta)$ & $\mu^{\prime}(t, \theta)$ \\
\hline Richards & $\frac{\alpha}{\left[1+k \times e^{-\gamma(t-\eta)}\right]^{1 / k}}$ & $\gamma \mu(t)\left[1-\left(\frac{\mu(t)}{\alpha}\right)^{k}\right]$ \\
3P logistic & $\frac{\alpha}{1+e^{-\gamma(t-\eta)}}$ & $\gamma \mu(t)\left[1-\frac{\mu(t)}{\alpha}\right]$ \\
5P logistic & $\alpha+\frac{\alpha_{0}-\alpha}{\left[1+\left(2^{1 / k}-1\right)(t / \eta)^{\gamma}\right]^{k}}$ & $-\frac{k \gamma}{t}[\mu(t)-\alpha]\left[1-\left(\frac{\mu(t)-\alpha}{\alpha_{0}-\alpha}\right)^{1 / k}\right]$ \\
Sigmoid Emax & $\alpha_{0}+\frac{t^{n}\left(\alpha-\alpha_{0}\right)}{t^{n}+\eta^{n}}$ & $\frac{n}{t}\left[\mu(t)-\alpha_{0}\right]\left[1-\frac{\mu(t)-\alpha_{0}}{\alpha-\alpha_{0}}\right]$ \\
4P Gompertz & $\alpha_{0}+\left(\alpha-\alpha_{0}\right) e^{-e^{-\gamma(t-\eta)}}$ & $-\gamma\left[\mu(t)-\alpha_{0}\right] \ln \left[\frac{\mu(t)-\alpha_{0}}{\alpha-\alpha_{0}}\right]$ \\
Weibull & $\alpha+\left(\alpha_{0}-\alpha\right) e^{-(t / \eta)^{\gamma}}$ & $\frac{\gamma}{t}[\mu(t)-\alpha] \ln \left[\frac{\mu(t)-\alpha}{\alpha_{0}-\alpha}\right]$ \\
\hline
\end{tabular}

(Burnham \& Anderson [19, 27], Claeskens \& Hjort [20]), which is used to account for model uncertainty by combining together the estimates from all the fitted models. Within the MA framework, one fits a set of $R$ candidate models, $g_{1}, g_{2}, \ldots, g_{R}$, to the data in order to obtain the parameter estimates from all models, $\widehat{\theta_{1}}, \widehat{\theta_{2}}, \ldots, \widehat{\theta_{R}}$. A post selection procedure $[20,23]$ implies that we first need to select the model with the best goodness-to-fit to the data, say $g_{\ell}$, and to estimate $\theta$ by $\widehat{\theta_{\ell}}$. The model selection can be 
based on an information criteria. However, this procedure does not take into account model uncertainty since the estimation is based on a single model. The MA techniques allow us to estimate the component in $\theta$ using information obtained from all fitted models and in that way to account for model uncertainty. Let us assume that the Akaike's Information Criterion (AIC [28]) is used for model selection. For a given set of $R$ candidate models, $g_{1}, g_{2}, \ldots, g_{R}$, Burnham $\&$ Anderson [27] proposed to rescale the AIC to

$\Delta \mathrm{AIC}_{i}=\mathrm{AIC}_{i}-\mathrm{AIC}_{\text {min }}, \quad i=1, \ldots, R$

here, $\mathrm{AIC}_{\min }$ is the smallest $\mathrm{AIC}$ value across the set of $R$ models. The AIC differences, $\Delta \mathrm{AIC}_{i}$, are interpreted as the information loss when model $g_{i}$, rather than the best model $g_{\min }$, is used to approximate the true and unknown model. Burnham \& Anderson [19] defined Akaike's weights as

$w_{i}(\mathrm{AIC})=\frac{\exp \left(-(1 / 2) \Delta \mathrm{AIC}_{i}\right)}{\sum_{i=1}^{R} \exp \left(-(1 / 2) \Delta \mathrm{AIC}_{i}\right)}$.

Akaike's weight $w_{i}$ (AIC) can be interpreted as the weight of evidence that model $g_{i}$ is the best model given a set of $R$ models and given that one of the models in the set must be the best model. The non-linear model with the highest Akaike's weight (i.e. the minimum AIC) is considered as the model with the best goodness-to-fit to the data.

Following Burnham \& Anderson [19], we can calculate the model averaged estimator for turning point $\left(\widehat{\eta}_{\mathrm{MA}}\right)$ and the final size of outbreak $\left(\widehat{\alpha}_{\mathrm{MA}}\right)$ as follow:

$\widehat{\eta}_{\mathrm{MA}}=\sum_{i=1}^{R} w_{i}(\mathrm{AIC}) \widehat{\eta}_{i}$,

$\widehat{\alpha}_{\mathrm{MA}}=\sum_{i=1}^{R} w_{i}(\mathrm{AIC}) \widehat{\alpha}_{i}$.

Here, $\widehat{\eta}_{i}$ and $\widehat{\alpha}_{i}$ are the parameter estimates for the turning point and final size of outbreak of $i$ th model, respectively. The estimators for variance for $\widehat{\eta}_{\mathrm{MA}}$ and $\widehat{\alpha}_{\mathrm{MA}}$ are given, respectively, by:

$\widehat{\operatorname{var}}\left(\widehat{\eta}_{\mathrm{MA}}\right)=\left[\sum_{i=1}^{R} w_{i}(\mathrm{AIC}) \sqrt{\widehat{\operatorname{var}}\left(\widehat{\eta}_{i} \mid M_{i}\right)+\left(\widehat{\eta}_{i}-\widehat{\eta}_{\mathrm{MA}}\right)^{2}}\right]^{2}$,

$\widehat{\operatorname{var}}\left(\widehat{\alpha}_{\mathrm{MA}}\right)=\left[\sum_{i=1}^{R} w_{i}(\mathrm{AIC}) \sqrt{\widehat{\operatorname{var}}\left(\widehat{\alpha}_{i} \mid M_{i}\right)+\left(\widehat{\alpha_{i}}-\widehat{\alpha}_{\mathrm{MA}}\right)^{2}}\right]^{2}$.

Note that one can replace the AIC by other information criteria such as Bayesian information criterion
(BIC), Kullback information criterion (KIC) and calculate the model's weight based on these criteria.

In addition, since the models' weights are based on the AIC (or any other information criterion) the MA approach described above ensure that the parameter estimates for the turning point and final size of the epidemic (and their standard errors) will be dominated by the model(s) with the best goodness-of-fit.

\section{RESULTS}

\section{Estimation of the final size and turning point using MA methods}

All non-linear models discussed above were fitted to the single-phase Zika outbreak in the cities of Bucaramanga, Cali, Cúcuta, and Neiva. The models were fitted to the weekly cumulative number of reported cases and the turning point and the final size were estimated for each city. The models were fitted using R software 3.3.1 [29], using the gnls function from package nlme [30].

Table 3 shows the parameter estimates, Akaike's information criteria and the Akaike's weights for all non-linear models used to calculate the model averaged estimates for the turning point and the final size of the outbreak. Figure 2 displays the cumulative predicted values and the incidence predicted values obtained for the fitted models to the complete data in each city, together with the observed values.

For Bucaramanga, the 5P logistic model $(\mathrm{AIC}=$ $348 \cdot 6)$, the Richards model (AIC $=361 \cdot 9)$, and the 3P logistic model (AIC $=397 \cdot 0)$ had lower AIC than the four-parameter (4P) Gompertz model (AIC = $424 \cdot 1)$, the sigmoid Emax model (AIC $=405 \cdot 5)$, and the Weibull model (AIC $=468 \cdot 3$ ). The MA final size estimate of the Zika outbreak is equal to 3700 cases (95\% CI 3632-3768) with a turning point close to the $19 \cdot 1$ weeks $(95 \%$ CI $18 \cdot 8-19 \cdot 3)$ after the begining of the outbreak. Note that the observed final size is equal to 3651 (Table 1). The 5P logistic model has an Akaike weight equal to 0.999 , while the weights obtained for the other models are relatively small which implies that the model average parameter estimated will be dominated by the 5P logistic model for this city.

Similar pattern was observed in Cali. The 5P logistic model (AIC $=451 \cdot 6)$, the Richards model $(\mathrm{AIC}=$ 494.9), and the 3P logistic model $(\mathrm{AIC}=511 \cdot 7)$ had lower AIC than the 4P Gompertz model (AIC = $527 \cdot 0)$, the sigmoid Emax $(\mathrm{AIC}=521 \cdot 1)$, and the 
Table 3. Parameter estimates for the turning point and final size of the epidemic obtained for the six non-linear model and their model average estimates per city

\begin{tabular}{|c|c|c|c|c|c|c|}
\hline City & Model & Turning point & Final size estimate & $k$ & AIC & Weight \\
\hline \multirow[t]{7}{*}{ Bucaramanga } & 3P logistic & $19 \cdot 0(18 \cdot 9,19 \cdot 1)$ & $3623(3596,3651)$ & \multirow{7}{*}{$0 \cdot 59(0 \cdot 48,0 \cdot 69)$} & $397 \cdot 0$ & $2.997 \times 10^{-11}$ \\
\hline & Richards & $18 \cdot 3(18 \cdot 1,18 \cdot 5)$ & $3680(3654,3705)$ & & $361 \cdot 9$ & $0 \cdot 001$ \\
\hline & 5P logistic & $19 \cdot 1(19 \cdot 0,19 \cdot 2)$ & $3700(3672,3729)$ & & $348 \cdot 6$ & 0.999 \\
\hline & Sigmoid Emax & $19 \cdot 3(19 \cdot 1,19 \cdot 5)$ & $3838(3785,3892)$ & & $405 \cdot 5$ & $4.435 \times 10^{-13}$ \\
\hline & 4P Gompertz & $16 \cdot 9(16 \cdot 8,17 \cdot 2)$ & $3789(3732,3845)$ & & $424 \cdot 1$ & $4.055 \times 10^{-17}$ \\
\hline & Weibull & $17 \cdot 6(17 \cdot 1,18 \cdot 0)$ & $4206(3994,4418)$ & & $468 \cdot 3$ & $9 \cdot 976 \times 10^{-27}$ \\
\hline & Model averaging & $19 \cdot 1(18 \cdot 8,19 \cdot 3)$ & $3700(3632,3768)$ & & & \\
\hline \multirow[t]{7}{*}{ Cali } & 3P logistic & $20 \cdot 3(20 \cdot 1,20 \cdot 5)$ & $12350(12204,12496)$ & \multirow{7}{*}{$0 \cdot 55(0 \cdot 38,0 \cdot 72)$} & $511 \cdot 7$ & $8.751 \mathrm{e} \times 10^{-14}$ \\
\hline & Richards & $19 \cdot 5(19 \cdot 1,19 \cdot 9)$ & $12645(12449,12841)$ & & $494 \cdot 9$ & $3.889 \times 10^{-10}$ \\
\hline & 5P logistic & $20 \cdot 3(20 \cdot 2,20 \cdot 4)$ & $12458(12333,12583)$ & & $451 \cdot 6$ & 1 \\
\hline & Sigmoid Emax & $20 \cdot 9(20 \cdot 6,21 \cdot 3)$ & $13510(13166,13854)$ & & $521 \cdot 1$ & $7 \cdot 846 \times 10^{-16}$ \\
\hline & 4P Gompertz & $18 \cdot 1(17 \cdot 8,18 \cdot 4)$ & $13216(12924,13508)$ & & $527 \cdot 0$ & $4 \cdot 101 \times 10^{-17}$ \\
\hline & Weibull & $19 \cdot 5(18 \cdot 5,20 \cdot 4)$ & $15927(14557,17298)$ & & $567 \cdot 2$ & $7 \cdot 590 \times 10^{-26}$ \\
\hline & Model averaging & $20 \cdot 3(19 \cdot 9,20 \cdot 8)$ & $12458(12023,12893)$ & & & \\
\hline \multirow[t]{7}{*}{ Cúcuta } & 3P logistic & $8 \cdot 9(8 \cdot 8,8 \cdot 9)$ & $4293(4255,4331)$ & \multirow{7}{*}{$1 \cdot 13(0 \cdot 86,1 \cdot 4)$} & $196 \cdot 8$ & $0 \cdot 370$ \\
\hline & Richards & $8 \cdot 9(8 \cdot 8,9 \cdot 2)$ & $4281(4237,4325)$ & & $197 \cdot 4$ & $0 \cdot 265$ \\
\hline & 5P logistic & $8 \cdot 9(8 \cdot 9,9 \cdot 0)$ & $4280(4231,4328)$ & & $196 \cdot 8$ & $0 \cdot 365$ \\
\hline & Sigmoid Emax & $8 \cdot 9(8 \cdot 8,9 \cdot 2)$ & $4433(4313,4554)$ & & $226 \cdot 9$ & $1.019 \times 10^{-7}$ \\
\hline & $4 \mathrm{P}$ Gompertz & $8 \cdot 0(7 \cdot 8,8 \cdot 2)$ & $4402(4277,4528)$ & & $233 \cdot 5$ & $4.024 \times 10^{-9}$ \\
\hline & Weibull & $8 \cdot 2(7 \cdot 8,8 \cdot 5)$ & $4673(4356,4991)$ & & $247 \cdot 9$ & $3 \cdot 010 \times 10^{-12}$ \\
\hline & Model averaging & $8 \cdot 9(8 \cdot 7,9 \cdot 1)$ & $4285(4199,4372)$ & & & \\
\hline \multirow{7}{*}{ Neiva } & 3P logistic & $9 \cdot 1(8 \cdot 9,9 \cdot 2)$ & $1912(1888,1936)$ & \multirow{7}{*}{$0 \cdot 49(0 \cdot 21,0 \cdot 78)$} & $184 \cdot 7$ & 0.003 \\
\hline & Richards & $8.7(8.5,8.9)$ & $1929(1905,1952)$ & & $176 \cdot 9$ & $0 \cdot 133$ \\
\hline & 5P logistic & $9 \cdot 1(8 \cdot 9,9 \cdot 1)$ & $1943(1916,1971)$ & & $175 \cdot 7$ & $0 \cdot 236$ \\
\hline & Sigmoid Emax & $9 \cdot 0(8 \cdot 9,9 \cdot 1)$ & $1945(1924,1967)$ & & $173 \cdot 8$ & 0.613 \\
\hline & 4P Gompertz & $8 \cdot 3(8 \cdot 2,8 \cdot 4)$ & $1946(1921,1972)$ & & $181 \cdot 3$ & 0.014 \\
\hline & Weibull & $8 \cdot 3(8 \cdot 2,8 \cdot 5)$ & $2008(1952,2064)$ & & $198 \cdot 3$ & $2 \cdot 884 \times 10^{-6}$ \\
\hline & Model averaging & $8 \cdot 9(8 \cdot 7,9 \cdot 3)$ & $1943(1913,1972)$ & & & \\
\hline
\end{tabular}

Weibull model $(\mathrm{AIC}=567 \cdot 2)$. The final size estimate for the Zika epidemic from the MA is equal to 12 458 cases (95\% CI $12023-12893)$, with an estimated turning point of $20 \cdot 3$ weeks ( $95 \%$ CI 19.9-20.8) after the Zika epidemic starts. As for Bucaramanga, in Cali, the Akaike's weight from the 5P logistic model is the highest and closed to 1, which implies that MA parameter estimates are mostly based on the 5P logistic model. The 5P logistic model is the model with the smallest AIC $(\mathrm{AIC}=196 \cdot 8)$ in Cúcuta as well. The 3P logistic (AIC $=196 \cdot 8)$ and Richards model $($ AIC $=197 \cdot 4)$ have similar AIC. Once again, the Weibull model has the highest information criteria $($ AIC $=247 \cdot 9)$. The MA estimate for final size is 4285 cases (95\% CI 4199-4372), with an estimated turning point of 8.93 weeks $(95 \%$ CI $8 \cdot 72-9 \cdot 15)$ after the outbreak begins. For this city Akaike's weights for the 3P logistic, the Richards and the 5P logistic models are equal to $0 \cdot 370,0 \cdot 265$, and $0 \cdot 365$, respectively. Hence, the MA estimates of final size and turning point are dominated by the parameter estimates obtained for these three models.

The model fit for the city of Neiva is sligthly different from the other cities reported above. Here, the models with the lowest AIC were the sigmoid Emax model $(\mathrm{AIC}=173 \cdot 8)$, the $5 \mathrm{P}$ logistic model $(\mathrm{AIC}=$ 175.7), and the Richards model (AIC $=176.9$ ), while the model with the highest AIC corresponded again to the Weibull model (AIC $=198 \cdot 3)$. The MA final size estimate for Neiva is equal to 1943 cases (95\% CI 1913-1972), and an estimated turning point of 8.9 weeks $(95 \%$ CI $8 \cdot 7-9 \cdot 3)$ after the outbreak starts. In Neiva, the highest Akaike's weight is for the sigmoid Emax model (0.613), followed by the 5P logistic $(0 \cdot 236)$ and Richards model $(0 \cdot 133)$.

\section{Real-time prediction}

The model average framework is particularly useful for real-time prediction since we use only part of the 

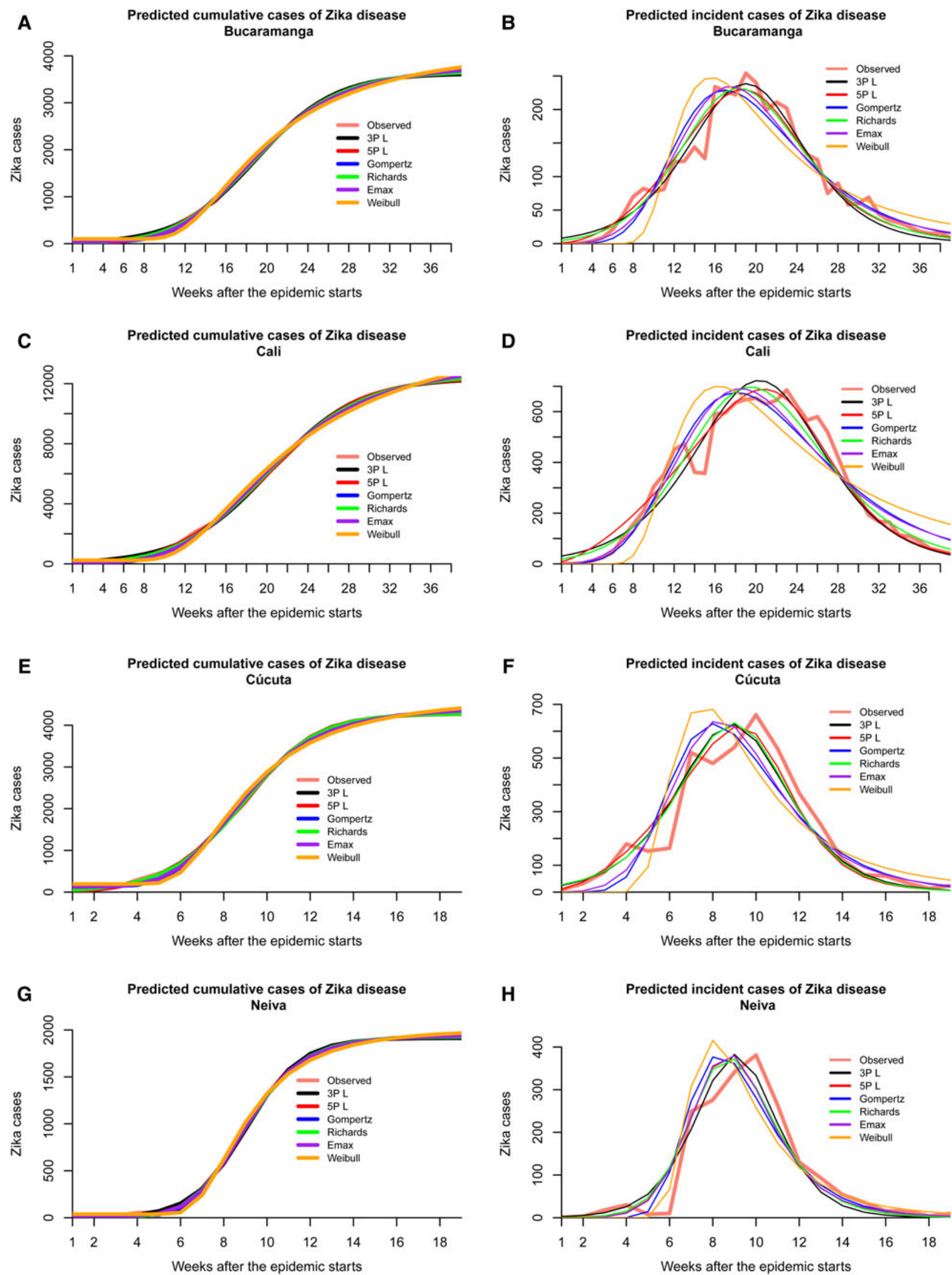

Fig. 2. Predicted cumulative and incidence cases based on six non-linear models for Zika outbreaks in four Colombian cities. Prediction is done when all data are used for the estimation of model parameters. 
data for long-term prediction. In this case, we do not want to base the estimation on a single model. Point estimates and 95\% CI obtained for all non-linear models and from the MA are presented in the supplementary appendix of the paper, for the turning point and final size of the outbreak in Bucaramanga, Cali, Cúcuta, and Neiva (Supplementary Tables S2-S5, respectively).

For Bucaramanga, the MA point estimate for the final size of the epidemic is 3452 cases $(95 \%$ CI 2982-3922), for the model fitted using the data of the first 26 weeks after the epidemic starts (Fig. 3d and Supplementary Table S2), while the observed final size of the outbreak is 3651 cases (Table 1). This implies that around 4 months before the end of the epidemic this valuable information could be available for the health authority. Furthermore, for the same estimation period, the MA estimate for the turning point becomes stable with a point estimate of 18.6 weeks $(17 \cdot 4,19 \cdot 8)$ (Fig. $3 \mathrm{~b}$ and Supplementary Table S2). These results indicate that the health authority could estimate the turning point of the outbreak about 6 weeks after its occurrence in Bucaramanga.

For Cali, the observed final size of the Zika epidemic is 12220 cases (Table 1). All the models overestimate the final size of the epidemic. The model average estimate stabilizes around the estimate of 12 560 cases (95\% CI 11848-13 272) for the models fitted to the period 1-33 weeks after the epidemic starts (i.e. 6 weeks before the end of the epidemic Fig. 3h and Supplementary Table S3). The MA estimate for the turning point obtained from the model fitted to the estimation period $1-33$ weeks is 22.5 weeks (95\% CI 21.5-19.4) (Fig. 3f and Supplementary Table S3).

For Cúcuta, The final size estimate from MA is stable from the model fitted for the estimation period 1-14 week, with a point estimate of 4308 cases $(95 \%$ CI 4039-4578) (Fig. 31 and Supplementary Table S4). The observed final size for Cúcuta is 4287 cases (Table 1). The turning point estimate from MA is equal to 8.9 weeks ( $95 \%$ CI $8 \cdot 6-9 \cdot 2)$, for the models fitted to the period $1-14$ weeks after the epidemic starts (i.e. 5 weeks before the end of the outbreak Fig. 3j and Supplementary Table S4).

For Neiva, the observed final size of the epidemic is 1940 cases (Table 1). The model average estimate for the final size of the epidemic is equal to 1899 cases (95\% CI 1742-2055) obtained for the model fitted to the data of the first 13 weeks of the outbreak (i.e. 6 weeks before the end of the outbreak Fig. 3p and Supplementary Table S5). The turning point estimate from MA becomes stable for the models fitted to period 1-13 week, showing a point estimate of 8.9 weeks $(95 \%$ CI $8 \cdot 5-9 \cdot 3$ ) (Fig. 3n and Supplementary Table S5).

In general, the models seem to fit the data for the shorter outbreaks (Cúcuta and Neiva) better than for the longer outbreaks (Bucaramanga and Cali). In addition, the Weibull model fits the data poorly for all cities. An elaborate discussion about the performance of the Weibull model in Bucaramanga and Cali is given in the supplementary material of the manuscript. The $95 \%$ CIs for the final size of the epidemic for all cities contain the observed final size, which implies that around 2-4 months before the end of the outbreak an accurate estimate for the outbreak's final size could be provided to the health authorities. The turning point estimates for Cúcuta and Neiva understimate the observed value by 1 week, but for Bucaramanga and Cali, the estimates are in line with the observed values, while the final size estimates estimate accurately the observed values for all cities (Fig. 3). The MA estimates for the turning point and final size of the epidemic were stable around the first $2 / 3$ of the total outbreak duration.

\section{DISCUSSION}

Modeling a single-wave outbreak requires to use a non-linear growth model in order to estimate the epidemiological parameters of interest. In this study we have shown that several non-linear models, the Richards, the 3P logistic, 5P logistic, the Weibull, sigmoid Emax and 4P Gompertz models, can be used to model the data. Alternatively, a model average technique that used a weighted parameter estimate, based on the model posterior probability, can be applied. In this paper we advocate the use of the MA technique since it does not ignore uncertainty related to model selection which is ignored when post selection inference and estimation is conducted.

Further, we have shown that the MA approach can be used in order to perform a real-time estimation for the turning point and prediction for the final size of the epidemic. We have shown that in the case of the Zika outbreak in 2015/2016 in Bucaramanga and Cali, an estimate with $95 \%$ CI that cover the observed final size could be given to the health authorities 4 months before the end of the epidemic, and for Cúcuta and Neiva accurate estimates for the final 
A Turning point estimates by model
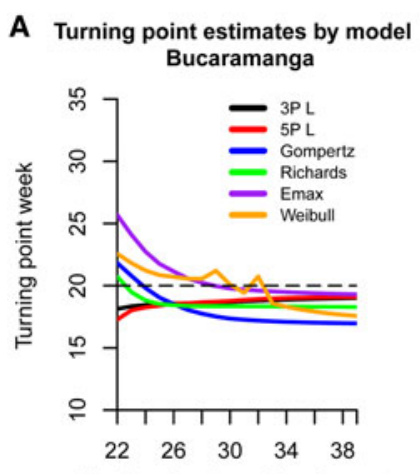

Weeks after the epidemic starts

E Turning point estimates by model Cali

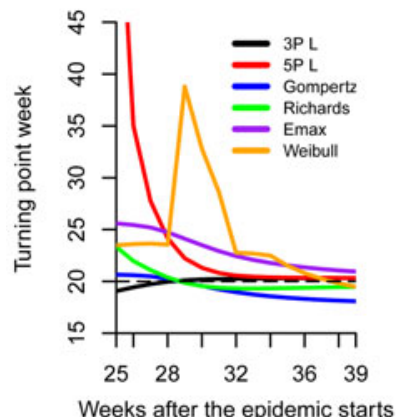

Weeks after the epidemic starts

I Turning point estimates by model Cúcuta

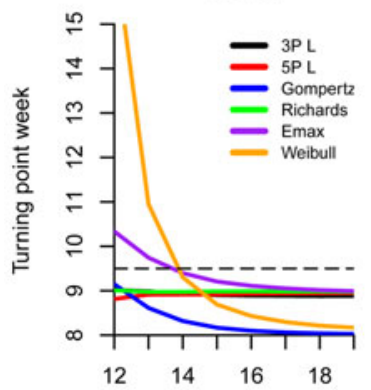

Weeks after the epidemic starts
B Turning point estimates by MA
Bucaramanga

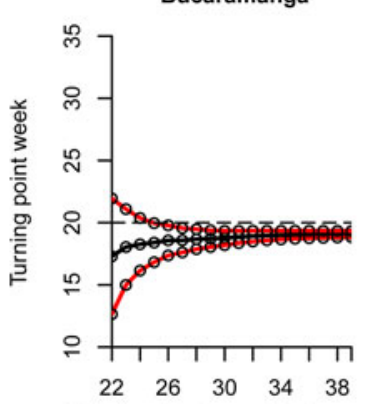

Weeks after the epidemic starts
C Final size estimates by model
Bucaramanga

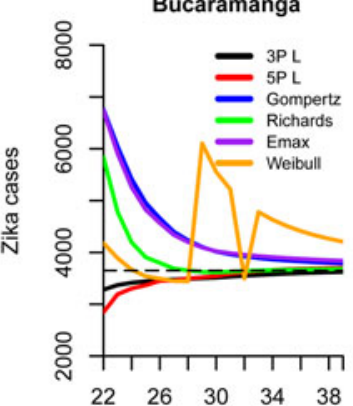

Weeks after the epidemic starts
D Final size estimates by MA

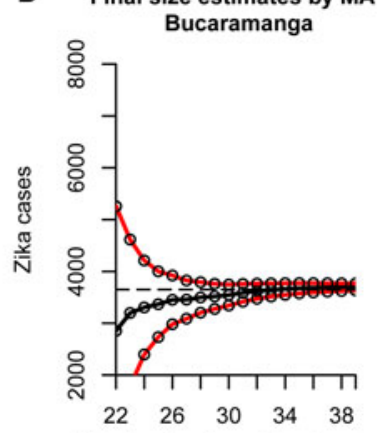

Weeks after the epidemic starts

F Turning point estimates by MA Cali

G Final size estimates by model Cali

H

Final size estimates by MA Cali
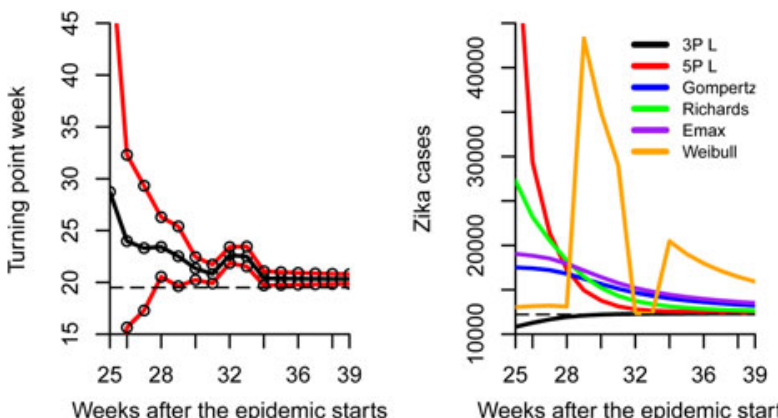

Weeks after the epidemic starts

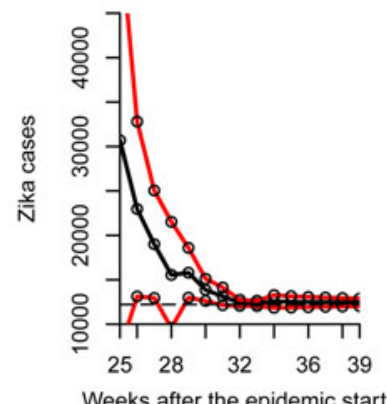

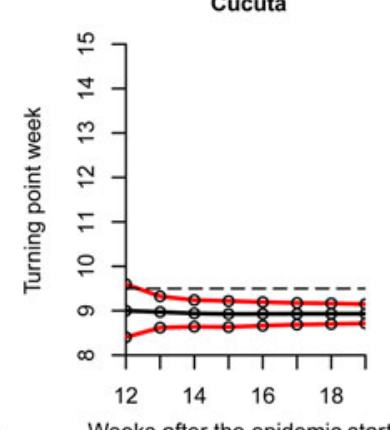

Weeks after the epidemic starts Cúcuta

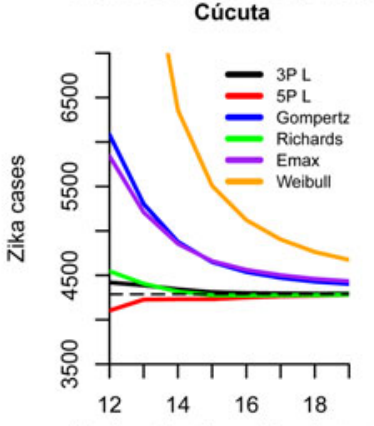

Weeks after the epidemic starts

K Final size estimates by model

M Turning point estimates by model $N$ Turning point estimates by MA
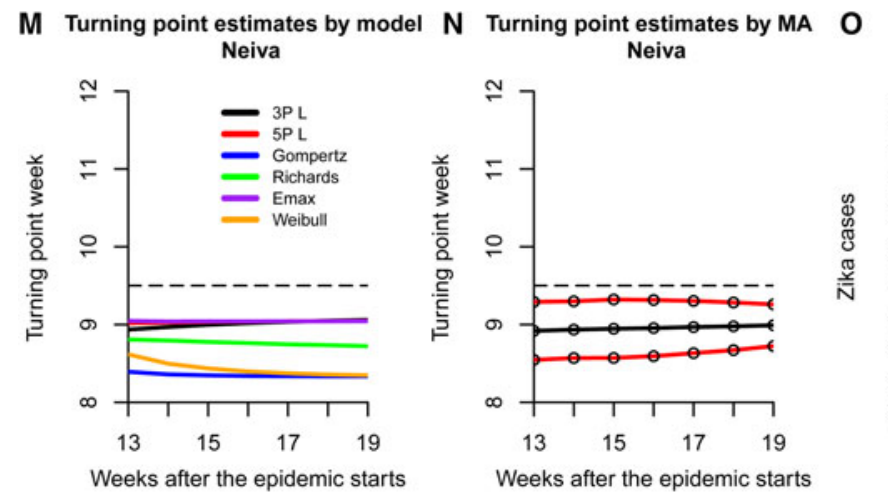
L Final size estimates by MA
Cúcuta

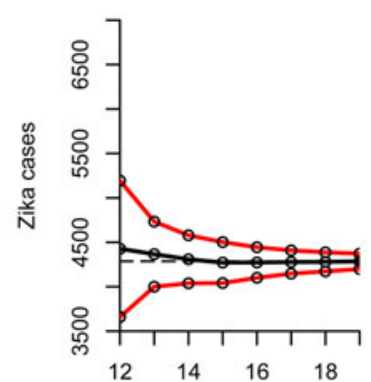

Weeks after the epidemic starts Neiva

$\mathbf{P}$

Final size estimates by MA Neiva

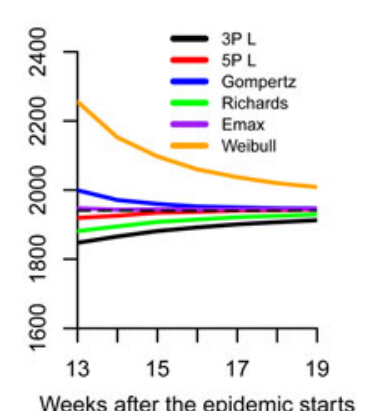

Fig. 3. Parameter estimates for the turning point and final size of the outbreak, from the non-linear models under study (point estimates), and from MA (point estimates and 95\% CI) per city. Dashed lines represent the observed values. The time scale in all figures present the last week in the estimation period. For example in panel a, 22 implies that the estimation period is $1-22$ weeks, etc. 
size could be provided 2 months before the end of the epidemic. Further, for a real-time prediction, the MA offers an attractive modeling approach since the data available for modeling represents only a part of the outbreak data (from the start to the time in which the real-time prediction is performed). Hence, to our opinion, taking into account several possible models is appropriate in this setting.

Since this study was conducted retrospectively, with data from routine surveillance system, potential biases could not be prevented. Some limitations include data quality associated with real-time modeling (as data are often subject to ongoing cleaning, correction, and reclassification of onset dates as further data become available) and reporting delays. Whether reporting delays or dates of reporting and date of onset were known, it would be possible to perform more realistic analyses that include only cases known about at the end of the most recent time period. This would likely make the models appear less attractive, but might provide a more realistic lower bound in terms of how quickly turning points can be identified.

Predicting the trend of an epidemic from limited data during early stages of the epidemic can be sometimes misleading. Nevertheless, early prediction of the magnitude of an epidemic outbreak is more important than retrospective studies [3]. The methodology proposed in the paper does not allow for a prediction of the turning point but only for the estimation. This is due to the fact that the non-linear models presented in the paper fit poorly the data in the initial stage of the outbreak, i.e., when the turning point of the outbreak can be predicted. As was observed in other attempts at real-time prediction, the forecast appears to be very vulnerable to the timing of predictions, especially during the early phase of epidemic [31]. As long as the data include this inflection point and a time interval shortly after, the curve fitting and predicting future case number will be reasonably accurate [3].

The MA modeling approach provides an attractive framework for real-time prediction since it takes into account a set of models and the real-time prediction is dominated by the model(s) with the best goodness-of-fit to the data. In the case of Bucaramanga and Cali, one model dominated the estimation and prediction (the 5P logistic), while in the case of Neiva and Cucuta the model average estimates was dominated by several models (3P logistic, Richard and 5P logistic in Cucuta with a combined weight $>0.999$ and Richard, 5P logistic, and sigmoid Emax with a combined weight of 0.982 in Neiva). This is the main advantage to use the MA framework for real-time prediction since, taking into account that only a part of the data is available and can be used for prediction, the estimation procedure is based on the best-fitted models.

The present study successfully offers a modeling strategy to implement real-time prediction of an epidemic in the midst of its course. The methodology discussed in this paper was developed for a single-wave outbreak. In future research we will extend the MA approach to a multi-wave outbreaks setting as well.

\section{DECLARATION OF INTEREST}

None.

\section{SUPPLEMENTARY MATERIAL}

The supplementary material for this article can be found at https://doi.org/10.1017/S0950268817001078

\section{ACKNOWLEDGEMENTS}

This research received no specific grant from any funding agency, commercial or not-for-profit sectors. D.A. Martínez Bello acknowledges the support of COLCIENCIAS by the grant 646-2014.

\section{REFERENCES}

1. Plourde AR, Bloch EM. A literature review of Zika virus. Emerging Infectious Diseases 2016; 22: 11851192.

2. Instituto Nacional de Salud. Reporte de notificación casos de Zika semana 32-2015 a semana 42-2016 [Zika case notification report, week 322015 - week 42 2016]. Colombia: Instituto Nacional de Salud [National Institute of Health], 2016. http://www.ins. gov.co/Noticias/ZIKA/Forms/AllItems.aspx.

3. Hsieh T, Lee J, Chang H. SARS epidemiology modeling. Emerging Infectious Diseases 2004; 10: 1165-1167.

4. Zhou G, Yan G. Severe acute respiratory syndrome epidemic in Asia. Emerging Infectious Diseases 2003; 9: 1608-1610.

5. Hsieh Y, Cheng Y. Real-time forecast of multi-wave epidemic outbreaks. Emerging Infectious Diseases 2006; 12: 122-127.

6. Richards $\mathbf{F}$. A flexible growth function for empirical use. Journal of Experimental Botany 1959; 10: 290-301.

7. Hsieh Y. Richards model: a simple procedure for real-time prediction of outbreak severity. In: Ma Z, Wu J, Zhoue Y, eds. Modeling and Dynamics of Infectious Diseases, Volume 11. Contemporary Applied Mathematics (CAM), Higher Education Press, 2009, pp. 216-236. DOI: 10.1142/9789814261265_0009. http://mail.cmu.edu. tw/hsieh/pdf/pub57.pdf. 
8. Hsieh Y, Ma S. Intervention measures, turning point, and reproduction number for dengue, Singapore, 2005. American Journal of Tropical Medicine and Hygiene 2009; 80: 66-71.

9. Hsieh Y, Chen C. Turning points, reproduction number, and impact of climatological events for multi-wave dengue outbreaks. Tropical Medicine and International Health 2009; 14: 628-638.

10. Hsieh Y, Arazoza H, Lounes R. Temporal trends and regional variability of 2001-2002 multiwave DENV-3 epidemic in Havana city: did hurricane Michelle contribute to its severity? Tropical Medicine and International Health 2013; 18: 830-838.

11. Hsieh Y-H et al. Early outbreak of 2009 influenza A (H1N1) in Mexico prior to identification of $\mathrm{pH} 1 \mathrm{~N} 1$ virus. PLoS ONE 2011; 6: e23853, 10: e0140810. DOI: 10.1371/journal.pone.0023853.

12. Hsieh Y-H. Temporal course of 2014 Ebola virus disease (EVD) outbreak in West Africa elucidated through morbidity and mortality data: a tale of three countries. PLOS ONE 2015; 10: e0140810. DOI: 10.1371/journal. pone.0140810.

13. Rozema E. Epidemic models for SARS and measles. The College Mathematics Journal 2007; 38: 246-259.

14. Tsoularis A, Wallace J. Analysis of logistic growth models. Mathematical Biosciences 2002; 179: 21-55.

15. Liao J, Liu R. Re-parameterization of five-parameter logistic function. Journal of Chemometrics 2009; 23: 248-253.

16. MacDougall J. Analysis of dose-response studies-Emax model. In: Ting $\mathrm{N}$, ed. Dose Finding in Drug Development. Statistics for Biology and Health. New York: Springer, 2006, pp. 127-145.

17. Wellock I, Emmans G, Kyriazakis I. Describing and predicting potential growth in the pig. Animal Science 2004; 78: 379-388.

18. Seber G, Wild C. Nonlinear Regression. New York: Wiley, 1989.

19. Burnham K, Anderson DR. Model Selection and Multimodel Inference: A Practical Information-theoretic Approach, 2nd edn. New York: Springer-Verlag, 2002.
20. Claeskens G, Hjort NL. Model Selection and Model Averaging. Cambridge: Cambridge University Press, 2008.

21. Posada D, Buckey TR. Model selection and model averaging in phylogenetics: advantages of Akaike Information Criterion and Bayesian approaches over likelihood ratio tests. Systematic Biology 2004; 53: 793-808.

22. Moon H et al. Model averaging using the Kullback Information Criterion in estimating effective doses for microbial infection and illness. Risk Analysis 2005; 25: $1147-1159$

23. Lin D et al. Modeling Dose-Response Microarray Data in Early Drug Development Experiments Using $R$. Berlin Heidelberg: Springer-Verlag, 2012.

24. Faes $\mathbf{C}$ et $\boldsymbol{a l}$. Model averaging using fractional polynomials to estimate a safe level of exposure. Risk Analysis 2007; 27: 111-123.

25. Jorgensen M. Fitting animal growth curves. The New Zealand Statistician 1981; 16: 5-15.

26. Wang X, Wu J, Yang Y. Richards model revisited: validation by and application to infection dynamics. Journal of Theoretical Biology 2012; 313: 12-19.

27. Burnham K, Anderson D. Multimodel inference: understanding AIC and BIC in model selection. Sociological Methods \& Research 2004; 33: 261-304.

28. Akaike H. Information theory and an extension of the maximum likelihood principle. In: Petrov BN, Csaki F, eds. Second International Symposium on Information Theory. Budapest: Akadémiai Kiadó, 1973, pp. 267-281.

29. R Core Team. R: A Language and Environment for Statistical Computing, R Foundation for Statistical Computing, Vienna, Austria, 2016. https://www. R-project.org/.

30. Pinheiro J et al. R Core Team, http://CRAN.R-project. org/package $=$ nlmenlme: Linear and Nonlinear Mixed Effects Models, $r$ package version 3.1-128, 2016. http://CRAN.R-project.org/package=nlme.

31. Nishiura H. Real-time forecasting of an epidemic using a discrete time stochastic model: a case study of pandemic influenza (h1n1-2009). BioMedical Engineering OnLine 2011; 10: 15. 